\title{
Desigualdade de renda e crescimento econômico: uma análise para o Brasil e suas regiões geográficas
}

\section{Income inequality and economic growth: an analysis for Brazil and its geographic regions}

\author{
Eliene de Sá Farias ${ }^{\text {ai }}$ \\ Orcid: https://orcid.org/0000-0001-9214-3591 \\ Evandro Camargos Teixeira ${ }^{\text {bii }}$ \\ Orcid: http://orcid.org/0000-0002-6470-2103
}

\begin{abstract}
Resumo
O presente estudo tem como objetivo analisar a relação entre crescimento econômico e desigualdade de renda no Brasil e para dois grupos de seus estados, mais desenvolvidos e menos desenvolvidos, entre os anos de 1997 e 2017, através da estimação de um painel dinâmico. Considerando o país como um todo, os principais resultados apontam que existe uma relação bicausal entre desigualdade de renda e crescimento econômico. Essa relação se mantém para os estados menos desenvolvidos, mas para os estados mais desenvolvidos apenas a desigualdade de renda precede o crescimento econômico. A partir dos resultados, sugere-se a formulação e implementação de políticas públicas que diminuam a desigualdade de renda no país, particularmente nos estados menos desenvolvidos.

Palavras Chaves: desigualdade de renda; crescimento econômico; dados em painel.
\end{abstract}

\begin{abstract}
The present study aims to analyze the relationship between economic growth and income inequality in Brazil and for two groups in their states, more developed and less developed, between the years 1997 and 2017, through the estimation of a dynamic panel. Considering the country as a whole, the main results indicate that there is a bicausal relationship between income inequality and economic growth. This relationship is maintained for the least developed states, but for the more developed states only income inequality precedes economic growth. From the results, it is suggested the formulation and implementation of public policies that reduce income inequality in the country, particularly in the least developed states.

Keywords: income inequality; economic growth; panel data.
\end{abstract}

\section{INTRODUÇÃO}

A discussão sobre a temática de desigualdade de renda contempla uma agenda global. De acordo com a FAO (2020), é necessário reduzir a desigualdade dentro e entre os países, sendo essa uma das metas para que seja alcançado o desenvolvimento sustentável. Nesse sentido, a desigualdade de renda entre todas as pessoas do mundo tem diminuído desde a década de 1990, mas dentro dos países,

\footnotetext{
a Universidade Federal de Viçosa, Brasil. E-mail: eliene.farias@ufv.br

${ }^{\mathrm{b}}$ Universidade Federal de Viçosa, Brasil. E-mail: evandro.teixeira@ufv.br
} 
principalmente naqueles em processo de desenvolvimento, a situação é diferente. $\mathrm{O}$ foco desses países, especialmente daqueles com elevadas disparidades na distribuição de renda, tem sido na redução da pobreza e aumento das oportunidades econômicas para toda população (WORLDBANK, 2020).

Ao longo do tempo, levantou-se o questionamento se a desigualdade de renda, que é mensurada principalmente através do índice de Gini $^{3}$, levava ao aumento ou redução do ritmo de crescimento econômico de um país. No caso da relação positiva entre desigualdade de renda e crescimento econômico, esta ocorre principalmente através da taxa de poupança, que é superior no caso da parcela da população mais rica em relação a mais pobre; e também via redistribuição de renda (SHIN,2012; BRIDA, CARRERA, SEGARRA, 2020). Por outro lado, a relação negativa se materializa particularmente através das imperfeições no mercado creditício, no qual os indivíduos com menor nível de renda possuem dificuldades de acesso ao crédito; e também através da instabilidade política e social, que pode ser notada na preferência do eleitor médio sem ativos por partidos políticos que priorizam os programas de redistribuição de renda (HELPMAN, 2004; TACHIBANAKI，2005，WEIL，2005; NEVES, SILVA, 2014).

As relações positivas ou negativas apontadas através dos canais mencionados podem ser visualizadas em diversos estudos internacionais. Alguns estudos atestam relação negativa entre crescimento econômico e desigualdade de renda (MURPHY et al., 1998; PEROTTI, 1993; ALESINA, RODRIK, 1994; PERSSON, TABELLINI, 1994; PEROTTI,1996; ALESINA, $\quad$ PEROTTI, 1996; ACEMOGLU, 1997; HELPMAN, 2004, TACHIBANAKI, 2005, KNOWLES, 2005; $\quad$ VOITCHOVSKY, 2005; SUKIASSVAN, 2007; CASTELLÓ, 2010;
CHAMBERS, KRAUSE, 2010; HERZER, VOLLMER,2012). Outros estudos concluem que existe relação positiva entre crescimento econômico e desigualdade de renda (OKUN, 1975; BOURGUIGNON,1990; BENABOU, 19996; LI E ZOU ,1998; AGHION, HOWITT, 1998, FORBES, 2000, BARRO,2000; CASTELLÓ,2010). Ainda, é possível verificar a existência de uma relação não monotônica com a forma de $\mathrm{U}$ invertido entre desigualdade de renda e crescimento econômico nos estudos de Chen (2003) e Bengoa e Robles (2005). A supracitada relação também pode ser inconclusiva (AMOS, 1998; BARRO, 2000; BANERJEE, DUFLO,2003; WEIL,2005, SHIN et al., 2009).

No que concerne o caso brasileiro, o país é considerado um dos mais desiguais do mundo. No período 2001-2004, a concentração de renda no Brasil caiu 4\%, o que se refletiu no declínio da pobreza e da pobreza extrema (BARROS, FOGUEL, ULYSSEA, 2006). No entanto, mais recentemente, entre 2017 e 2018, apenas $2,7 \%$ das famílias brasileiras concentravam quase $20 \%$ de toda renda recebida no país. Segundo pesquisa do IBGE para o período mencionado, $2,7 \%$ do total de famílias, ou seja, 1,8 milhão possuía rendimento superior a R\$23.850,00. Por outro lado, $23,9 \%$, ou seja, 16,4 milhões de famílias, recebiam somente até $\mathrm{R} \$ 1.908,00$ (OHANA, 2019).

Além disso, em termos regionais, de acordo com dados da Pesquisa Nacional por Amostra de Domicílios PNAD contínua do IBGE (2019), na região Nordeste a desigualdade aumentou, conforme denotado pelo índice de Gini, que passou de 0,545 em 2018 para 0,559 em 2019. Isso pode ser explicado pelo fato que na região houve grande elevação na renda da população $1 \%$ mais rica, cerca de $14,9 \%$, com concomitante diminuição de aproximadamente $5 \%$ entre os $10 \%$ mais pobres, evidenciando maior concentração

\footnotetext{
${ }^{3}$ Mede a concentração de renda, sendo que quanto mais próximo seu valor estiver de 1, maior a desigualdade; e quanto mais próximo de 0 , menor a desigualdade.
} 
de renda. Por sua vez, a região Norte denotou a maior queda no referido índice entre as regiões brasileiras, caindo de 0,551 em 2018 para 0,537 em 2019 (IBGE, 2019). No que tange à região Sudeste, esta também evidenciou importante redução da concentração de renda entre os referidos anos, dado que o valor do índice de Gini caiu de 0,533 para 0,527 .

A supracitada elevada concentração de renda brasileira estimulou a elaboração de trabalhos, relacionando-a com o ritmo de crescimento econômico, encontrando os mais diversos resultados. Nesse sentido, o estudo de Jacinto e Tejada (2009) avaliou a relação entre desigualdade de renda e crescimento econômico nos municípios do Nordeste entre os anos 1970 e 1991, apontando a existência de uma curva de Kuznets ${ }^{4}$. Por sua vez, Castro e Júnior (2007) avaliaram a referida relação para o país como um todo por meio de uma análise não-linear, atestando uma relação negativa. Já Cruz, Teixeira e Monte-Mor (2015) demonstraram que a desigualdade de renda no Brasil possui efeito positivo sobre o crescimento econômico, contrariando as conclusões de Santos, Cunha e Gadelha (2016) a respeito da existência de uma relação bicausal.

Considerando a supracitada elevada desigualdade de renda brasileira, caracterizada sobretudo pelas discrepâncias regionais, o objetivo deste trabalho consiste em analisar a relação entre desigualdade de renda e crescimento econômico para o período 1997-2017 no Brasil. O estudo aborda essa relação através de um painel dinâmico, que considera as dissimilaridades regionais brasileiras. Para essa finalidade, além de analisar o país como um todo, o estudo divide os estados brasileiros em dois grupos: mais desenvolvidos e menos desenvolvidos. Essa distinção leva em consideração as condições socioeconômicas dos estados, de acordo com variáveis como taxa de alfabetização, índice de desenvolvimento humano, índice de Gini e crescimento do Produto Interno Bruto (PIB) (BERTUSSI; ELLERY JUNIOR, 2012). O primeiro grupo corresponde às regiões consideradas menos desenvolvidas, representadas pelos estados da região Norte, Nordeste e Centro-Oeste, incluindo o Distrito Federal. $\mathrm{O}$ segundo grupo contempla as regiões consideradas mais desenvolvidas, Sudeste e Sul. O trabalho contribuiu na literatura ao estender a análise realizada em Santos, Cunha e Gadelha (2016), ao atestar a causalidade existente entre distribuição de renda e crescimento econômico para o Brasil e grupos de estados, além de considerar um período mais recente.

Além desta introdução, o trabalho está dividido em outras quatro seções. A segunda corresponde à discussão empírica que permeia a relação entre desigualdade de renda e crescimento econômico. A terceira engloba os procedimentos metodológicos adotados. Posteriormente, são analisados os resultados, e por fim, têm-se as considerações finais.

\section{EVIDÊNCIAS EMPÍRICAS ACERCA DA RELAÇÃO ENTRE DESIGUALDADE DE RENDA E CRESCIMENTO ECONÔMICO}

Como já ressaltado, a literatura que especifica a relação entre desigualdade de renda e crescimento econômico apresenta distintos resultados. Alguns estudos que denotam a existência de relação positiva concluem que a mesma se dá através da taxa de poupança (BRIDA, CARRERA, SEGARRA, 2020). Levando em consideração que a taxa de poupança da parcela da população mais rica é superior em relação à população mais pobre, a redistribuição de renda reduz o ritmo de crescimento econômico (SHIN, 2012).

A referida relação positiva entre desigualdade de renda e crescimento

\footnotetext{
${ }^{4}$ Nos estágios iniciais do crescimento econômico, a desigualdade de renda aumenta, mas ao longo do tempo essa se reduz.
} 
econômico também pode ocorrer em função da redistribuição de renda propriamente dita. Nesse caso, a redistribuição de renda pode reduzir o incentivo para que a parcela da população que já detinha maior nível de capital oferte mais trabalho, o que conduz a um declínio no ritmo de crescimento econômico (SHIN, 2012).

Por outro lado, há um conjunto de trabalhos que determinaram que a relação entre desigualdade de renda e crescimento econômico é negativa, dadas as imperfeições no mercado de crédito nos países em desenvolvimento (HELPMAN, 2004; TACHIBANAKI,2005, WEIL, 2005). Assim, como grande parte da população não tem condições de investir, tanto em capital físico como capital humano, pois não tem acesso a crédito, ocorre declínio do ritmo de crescimento econômico (BRIDA, CARRERA, SEGARRA, 2020).

Outro canal pelo qual a desigualdade de renda compromete o ritmo de crescimento econômico se dá através da instabilidade política e social (NEVES, SILVA,2014). Além desse, a supracitada relação negativa entre desigualdade de renda e crescimento econômico também tem como hipótese a abordagem da economia política. Nesse caso, as políticas redistributivas do governo, que objetivam melhorar o acesso da população mais pobre aos recursos econômicos, podem ter como contrapartida elevação dos impostos, comprometendo o ritmo de crescimento econômico (ALESINA, RODRICK, 1994; PERSSON, TABELLINI, 1994).

Outra evidência que aponta para a existência de relação negativa entre desigualdade de renda e crescimento econômico envolve os conflitos sociais (ALESINA, PEROTTI, 1994). Tais conflitos podem ser visualizados através da elevação dos crimes e atividades ilegais, que ameaçam os investimentos e os direitos de propriedade, principalmente nos países em desenvolvimento.

Kuznets (1995) encontra uma relação diferente entre desigualdade de renda e crescimento econômico, que parece assumir a forma de "U invertido". O autor observou que no início do processo de crescimento econômico a distribuição de renda muda de relativa igualdade para desigualdade, e posteriormente volta a ser relativamente igualitária à medida que $\mathrm{o}$ país cresce.

Como verificado até aqui, a literatura evidencia algumas possibilidades em termos da relação entre desigualdade de renda e crescimento econômico. Para Barro (2000), tais relações variam de acordo com nível de desenvolvimento econômico dos países. Segundo o autor, nos países pobres, a desigualdade de renda retarda o ritmo de crescimento econômico. Por outro lado, a desigualdade de renda incentiva $o$ crescimento econômico em um país desenvolvido.

Shin (2012), ao analisar de forma teórica a relação entre desigualdade de renda e crescimento econômico, sustentou a hipótese de Barro (2000). De acordo com Shin (2012), a depender do nível de desenvolvimento econômico do país, ambos resultados são possíveis, ou seja, maior desigualdade de renda pode retardar o crescimento econômico no estágio inicial de desenvolvimento e incentivar o crescimento econômico em um patamar considerado quase estável.

Empiricamente, a maioria dos estudos demonstra a existência de relação negativa entre crescimento econômico e desigualdade de renda nos países em desenvolvimento (KNOWLES, 2005 ; VOITCHOVSKY，2005; CASTELLÓ, 2010 ; CHAMBERS e KRAUSE, 2010 ; KHALIFA e EL HAG, 2010 ; HERZER e VOLLMER, 2012). Essa relação também se mostrou não significativa para alguns estudos aplicados a países em 
desenvolvimento (PEROTTI, 1996; BARRO, 2000).

No que tange à literatura brasileira, os resultados são diversos. O estudo de Castro e Júnior (2007) avaliou a relação entre desigualdade de renda e crescimento econômico no país e os resultados vão ao encontro da maior parte da literatura internacional para países em desenvolvimento, ou seja, a relação entre desigualdade de renda e crescimento econômico é negativa.

Por sua vez, Jacinto e Tejada (2009) analisaram a referida relação para os municípios da região Nordeste brasileira entre 1970 e 1991, encontrando resultados que denotam a existência de "U invertido", como em Kuznets (1995). Já o trabalho de Cruz, Teixeira e Monte-Mor (2015) demonstrou que a desigualdade possui efeito positivo sobre o crescimento econômico brasileiro no período 19902009, quando são observados níveis de PIB per capita mais elevados, enquanto o efeito torna-se negativo quando os níveis de PIB per capita são mais baixos. Resultado distinto foi encontrado por Santos, Cunha e Gadelha (2016). Os autores utilizaram o modelo VEC e ADLs para o período 19762012 e verificaram que desigualdade de renda e crescimento econômico apresentam uma relação bicausal no sentido de Granger.

\section{METODOLOGIA}

\subsection{Modelo econométrico}

A relação de causalidade estatística entre desigualdade de renda e crescimento econômico pode ser realizada por meio de diferentes abordagens com a disposição dos dados em painel. Estas possuem divergências em termos das suposições sobre a homogeneidade dos coeficientes considerados entre os estados do Brasil. Uma dessas abordagens consiste em tratar os dados em painel como um conjunto de dados empilhados e executar o teste de causalidade de Granger na forma padrão. Dessa forma, os dados em seção transversal não terão valores defasados da próxima seção transversal, de modo que assume-se que todos os coeficientes sejam iguais em todas as transversais. No entanto, o presente estudo utiliza a abordagem de Dumitrescu e Hurlin (2012), que possui uma suposição completamente distinta, onde os coeficientes são diferentes nas seções transversais. Para que seja realizada essa estimativa, os valores de desigualdade de renda e taxa de crescimento do PIB per capita foram transformados em números índices, o que permite a padronização dessas medidas.

Em seguida, para avaliar o efeito da desigualdade de renda sobre a taxa de crescimento econômico é utilizado um painel dinâmico dos estados brasileiros e Distrito Federal para o período 1997-2017. O período de análise escolhido teve como critério a disponibilidade de dados. Por sua vez, o modelo econométrico usado será o método dos momentos generalizados, System GMM, tal como expresso nos estudos de Arellano e Bond (1991), Arellano e Bover (1995) e Blundell e Bond (1998). As vantagens em sua utilização comparadas aos demais métodos envolvem a ausência de viés, derivados dos efeitos fixos temporalmente invariantes, e a consistência do estimador diante da possível relação de endogeneidade.

O painel dinâmico proposto por Arellano e Bond (1991), denominado difference GMM, possui a equação diferenciada em primeira ordem, e, portanto, é livre de efeitos fixos. Porém, como as variáveis explicativas tornam-se instrumentos fracos, tem-se um problema na utilização desse estimador. Isso porque se os instrumentos são fracos assintoticamente, o resultado consiste em um aumento da variância dos coeficientes, e para o caso específico de amostras pequenas, os coeficientes podem ser viesados.

A fim de reduzir o viés potencial e os problemas de inconsistência, Arellano e Bover (1995) e Blundell e Bond (1998) propuseram um momento adicional ao GMM, de forma que tem-se um sistema de 
regressões em diferenças e níveis, ao adicionarem ao difference GMM a equação original em nível, aumentando a eficiência, em função da presença de mais instrumentos. $\mathrm{O}$ sistema com duas equações foi denominado de System GMM. A ideia básica desse método consiste em combinar equações em primeiras diferenças e em nível. Na equação em diferença, os níveis defasados são os instrumentos das primeiras diferenças. Por sua vez, os instrumentos usados na equação em nível são as primeiras diferenças defasadas das séries. A equação do modelo proposto no presente estudo é dada por:

$$
\begin{aligned}
& \text { TXCRPIBCA } A_{i t}=\beta_{1} \text { TXCRPIBCA }_{i, t-1}+\beta_{2} I T_{i t} / P I B+\beta_{3} I N D I C E G I N I_{i t}+\beta_{4} \text { RENDA }_{i t}+\beta_{5} \text { TAXAALF }_{i t}+\beta_{6} A B C \\
& +n_{i}+v_{i t}
\end{aligned}
$$

Da equação (1), tem-se que i são os 26 estados brasileiros e Distrito Federal; t são os anos da análise, no caso 1997 a 2017. TXCRPIBCA corresponde à taxa de crescimento anual do PIB per capita. É importante salientar que as variáveis explicativas tiveram como critério de seleção a literatura concernente ao tema. A seguir, tem-se uma breve discussão da justificativa das variáveis explicativas inseridas. Nesse sentido, a principal variável explicativa do estudo consiste na desigualdade de renda, representada pelo índice de Gini. A forma como essa variável pode influenciar a taxa de crescimento anual do PIB per capita foi explicitada na seção anterior.

Os demais controles adicionados são apresentados a seguir. A variável IT/PIB corresponde à participação do investimento total efetuado pelo governo como proporção do PIB de cada estado e Distrito Federal. Estudos que analisam a conexão entre investimentos públicos e crescimento econômico é vasta na literatura. Um dos inspiradores dessa conexão foi o estudo de Aschauer (1989). A partir deles, é possível verificar diversos outros estudos que associam de forma sugestiva que acréscimos no crescimento econômico decorrem de investimentos públicos (EASTERLY, REBELO,1993; POSE, TSELIOS, PYCHARIS,2012, PALEI,2015). Devarajan et al. (1996) inclusive caracterizam o investimento público produtivo como aquele que promove aumento da taxa de crescimento de um país.
Renda média domiciliar $(R E N D A)$ é definida como o somatório dos rendimentos de todas as fontes dos membros do domicílio dividida pelo número dos membros do domicílio. Como a renda é restringida ao âmbito domiciliar, ela reflete o montante de recursos que uma determinada família possui para seu consumo. A finalidade desse controle é indicar como o consumo domiciliar estadual pode influenciar o crescimento econômico.

Taxa de alfabetização (TAXAALF) foi inserida para captar o nível de capital humano. Assim, o maior nível de escolaridade implica em indivíduos com maior habilidade no mercado de trabalho (DE SOUZA, 1999). Geralmente, se os indivíduos apresentam níveis baixos de qualificação em termos de educação, o resultado esperado é maior probabilidade de estarem desempregados, o que está relacionado consequentemente a um nível de renda mais baixo (SATTI et al., 2016).

No que tange à variável Abertura comercial $(A B C)$, a literatura aponta que um país pode ter diversos benefícios com a abertura comercial. Por exemplo, é bem discutido que as exportações de um país provocam efeito no crescimento econômico (BALASSA, 1978; DODARO, 1991; HERRERIAS; ORTS, 2010). Para países em desenvolvimento, como o Brasil, os benefícios da abertura comercial foram observados a partir dos anos oitenta (BURANGE, RANADIVE, KARNIK, 2019). Para os autores, as medidas voltadas para fomentar as exportações atuaram na 
alocação de recursos domésticos e na busca por produção eficiente, resultando em aumento da produtividade, indústrias com vantagens comparativas, e busca por inovações. Por isso, espera-se que maior abertura comercial leve a um aumento do crescimento econômico.

Por fim, é importante definir que $n_{i}$ é um fator específico não observável; e $v_{i t}$ é o resíduo.

Para testar a consistência do estimador do System GMM, são considerados dois tipos de testes. Para atestar a validade das variáveis utilizadas como instrumentos, o Teste de Sargan será aplicado, que corresponde a um teste de restrição de sobre-identificação. A hipótese nula desse teste é de que os instrumentos usados são válidos, implicando que os mesmos não são correlacionados com o termo de erro. O segundo teste é o de ausência de autocorrelação no termo de erro. Isso é, o teste tem como hipótese nula a ausência de correlação serial.

Para maior robustez dos resultados, e dado que o Brasil possui regiões com diferentes níveis de desenvolvimento, os estados serão separados em dois grupos. Tais grupos se diferenciam de acordo com o nível de desenvolvimento, no caso mais desenvolvidos e menos desenvolvidos.

\subsection{Base de dados}

O PIB corrente (milhões de reais) e o índice de Gini tiveram como fonte o
Banco Sidra do Instituto Brasileiro de Geografia e Estatística (IBGE), das tabelas correspondentes ao Produto Interno Bruto dos Municípios, para o período 2002-2017. As observações para essas variáveis no período 1997-2001 foram retiradas do Datasus (indicadores sociais). Os dados do quantitativo da população também tiveram como fonte o IBGE.

Para o investimento total (R\$), foram utilizados os dados disponibilizados pelo Ministério da Fazenda- Secretaria do Tesouro Nacional. Por sua vez, o rendimento médio domiciliar nominal tem como fonte a Pesquisa Nacional por Amostra de Domicílios (PNAD) e a PNAD contínua do Banco Sidra do IBGE.

Já, a taxa de alfabetização (\%) foi retirada dos dados do DATASUS (indicadores socioeconômicos). No que tange à variável de abertura comercial $(A B C)$, esta consiste na soma das exportações e importações dividida pelo PIB. Os dados de exportação (US\$) e importação (US\$) free on board utilizados referem-se aos dados disponibilizados no site do Ministério da Economia, indústria, comércio exterior e serviços (MDIC).

As variáveis monetárias foram deflacionadas de acordo com o Índice Nacional de Preços ao Consumidor Amplo (IPCA) do IBGE, tomando como base o ano de 2017. A Tabela 1, abaixo, apresenta as estatísticas descritivas das variáveis utilizadas em painel para todos os estados e Distrito Federal.

Tabela 1 - Estatísticas descritivas.

\begin{tabular}{lcccc}
\hline Variável & Média & Desvio Padrão & Valor mínimo & Valor máximo \\
\hline TXCRPIBCA & 1.304151 & 1.286397 & -0.9064666 & 11.21762 \\
INDICE DE GINI & 0.7443015 & 0.0808134 & 0.4439 & 0.907582 \\
RENDA & 419.1728 & 333.9959 & 65.4712 & 2474.988 \\
TAXAALF & 87.19049 & 7.520584 & 63.75 & 97.5 \\
IT/PIB & 159580.2 & 1681790 & 0 & $2.98 \mathrm{e}+07$ \\
ABC & 0.1118997 & 0.8468095 & $1.00 \mathrm{e}-09$ & 16.77835 \\
\hline
\end{tabular}

TXCRPIBCA é a taxa de crescimento anual do pib per capita; renda é a renda média domiciliar; TAXAALF é a taxa de alfabetização; IT/PIB é o investimento total como proporção do pib de cada estado; ABC é a abertura comercial.

Fonte: Elaboração própria. 
$\mathrm{Na}$ média geral, o crescimento do PIB per capita dos estados brasileiros de 1997 a 2017 foi de 1.304151, enquanto que a desigualdade de renda, medida pelo índice de Gini, foi de 0.7443015 no período. Com relação às variáveis de controle inseridas, na média geral, no período 1997- 2017, a renda domiciliar foi de $\mathrm{R} \$ 419.1728$, a taxa de alfabetização de $87.19 \%$, e o investimento total/PIB de R\$159580.2

Para se ter uma dimensão da taxa de crescimento do PIB per capita e da desigualdade de renda, no total (país como um todo), e pela divisão dos estados de acordo com o nível de desenvolvimento, tem-se a Figura 1 a seguir.

Figura 1 - Média geral da taxa de crescimento anual do PIB per capita e do índice de Gini no período $1997-$ 2017.

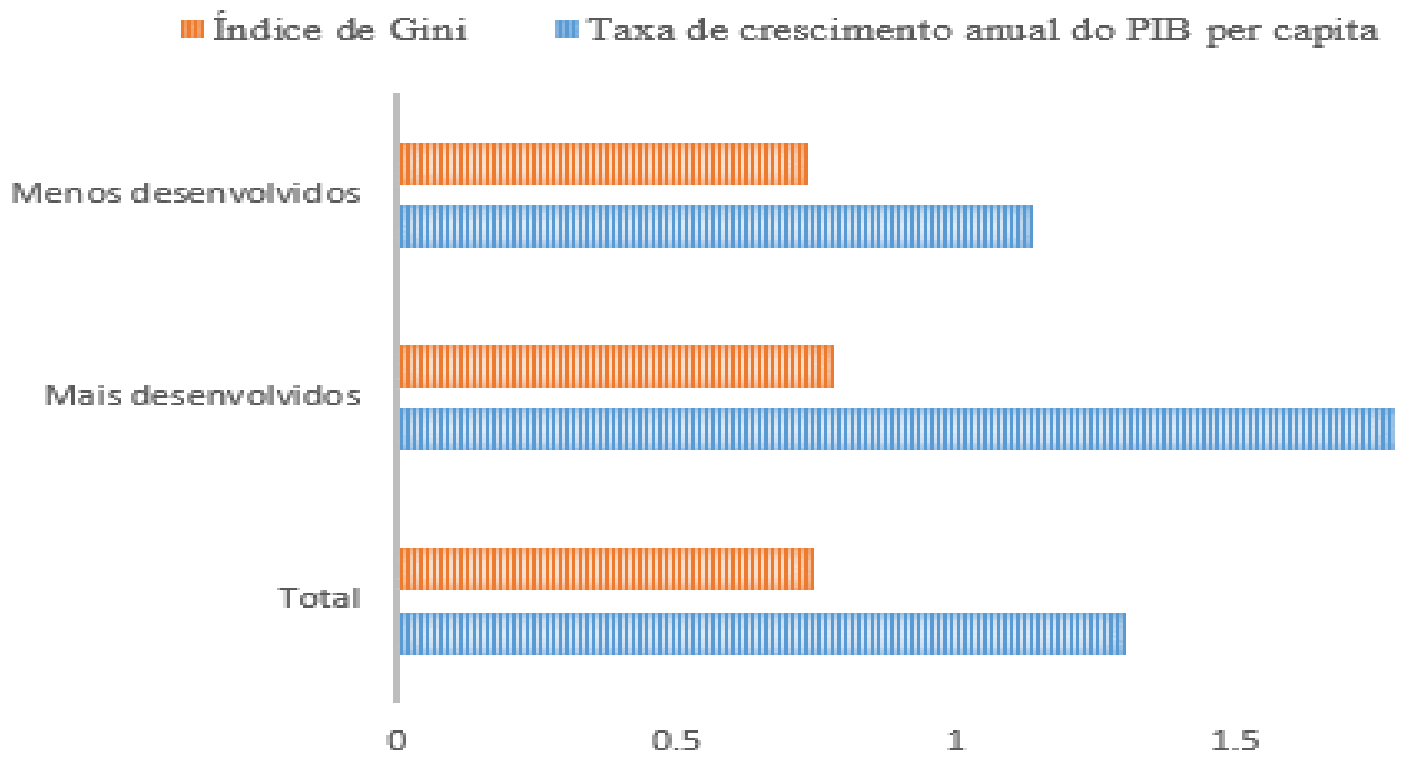

Fonte: Elaboração própria.

Como demonstra a Figura 1, no Brasil como um todo, a média da taxa de crescimento anual do PIB per capita entre 1997 e 2017 foi de aproximadamente $1,30 \%$, e a desigualdade de renda apresentou média de 0,74 . Os estados mais desenvolvidos apresentaram média mais elevada de crescimento econômico, quando comparados aos estados menos desenvolvidos. Nesse sentido, para o referido grupo de estados, no período 19972017, a média da taxa de crescimento anual do PIB per capita foi de cerca de $1,78 \%$ e a desigualdade de renda foi de aproximadamente 0,78 . Por outro lado, nos estados menos desenvolvidos, em média, para o período assinalado, a taxa de crescimento anual do PIB per capita foi de cerca de $1,13 \%$ e a desigualdade de renda foi de aproximadamente 0,73 , pouco inferior aos estados mais desenvolvidos.

\section{RESULTADOS E DISCUSSÃO}

Os resultados do teste de causalidade utilizado de Dumitrescu e Hurlin (2012), no qual assume-se uma abordagem mais realista, considerando que todos os estados possuem comportamentos distintos, são apresentados na Tabela 2 . Considera-se que Y per capita corresponde à taxa de crescimento do $\mathrm{PIB}$ per capita $\mathrm{e} \mathrm{G}$ à desigualdade de renda. 
Tabela 2 - Resultado do teste de Causalidade de Granger em painel

\begin{tabular}{|c|c|c|c|}
\hline \multicolumn{4}{|l|}{ Total } \\
\hline Hipótese nula & W-stat. & Zbar-Stat & Prob. \\
\hline Y per capita Granger não causa $G$ & 11.0285 & 29.352 & $0.0000 * * *$ \\
\hline G Granger não causa Y per capita & 3.03209 & 5.65231 & $0.0000 * *$ \\
\hline \multicolumn{4}{|l|}{ Mais Desenvolvidos } \\
\hline Y per capita Granger não causa $G$ & 0.44122 & -1.03190 & 0.3021 \\
\hline G Granger não causa Y per capita & 2.43934 & 1.98348 & $0.0473 * *$ \\
\hline \multicolumn{4}{|l|}{ Menos desenvolvidos } \\
\hline Y per capita Granger não causa $\mathrm{G}$ & 14.7341 & 34.7148 & $0.0000 * * *$ \\
\hline G Granger não causa Y per capita & 3.23956 & 5.3934 & $0.0000 * *$ \\
\hline
\end{tabular}

*** significativo a $1 \%, * *$ significativo a 5\%, $*$ significativo a $10 \%$. O teste considerou 1 defasagem. Fonte: Elaboração própria, resultados da pesquisa.

De acordo com o referido teste, a hipótese de que crescimento econômico não causa Granger desigualdade de renda é rejeitada. Isso significa que para a amostra que contempla o país como um todo, o crescimento econômico causa, no sentido de Granger, a desigualdade de renda. É possível observar que essa relação é bicausal, ou seja, a desigualdade de renda também impacta o crescimento econômico. Esse resultado é similar ao verificado no estudo de Santos, Cunha e Gadelha (2016), que analisou essa relação para o Brasil no período 1976-2012, utilizando dados de séries temporais. Segundo os autores, o crescimento econômico afeta de forma positiva a distribuição de renda, e esta impacta negativamente $\mathrm{o}$ crescimento econômico. Os resultados do trabalho de Souza (2019) também evidenciaram que elevações nas taxas de crescimento econômico entre os anos de 2004 e 2014 implicaram em quedas do nível de pobreza nos meios urbano e rural brasileiro.

Por outro lado, para os estados mais desenvolvidos, observou-se que a hipótese do teste é rejeitada apenas no sentido de que a desigualdade Granger não causa a taxa de crescimento do PIB per capita. Isso demonstra que para os referidos estados, a relação entre taxa de crescimento econômico e desigualdade de renda é unicausal. Nesse caso, só existe causalidade no sentido de Granger da desigualdade impactando o crescimento econômico. No entanto, para a amostra de estados menos desenvolvidos, os resultados foram semelhantes à amostra total, que considera o país como um todo, sinalizando que há relação bicausal entre desigualdade de renda e crescimento econômico.

Ademais, os resultados da precedência temporal entre crescimento econômico e desigualdade de renda parece indicar uma relação de simultaneidade entre essas variáveis, o que é controlado através da estimação do painel dinâmico. Assim, a seguir, tem-se a Tabela 3 com os resultados da estimação do modelo econométrico com dados em painel dinâmico, que tem como objetivo central analisar a relação entre desigualdade de renda e crescimento econômico.

Tabela 3 - Resultados da estimação do painel dinâmico.

\begin{tabular}{lccc}
\hline VARIÁVEL DEPENDENTE: & $T X C R P I B C A-(\mathrm{Y})$ & & \\
\hline VARIÁVEIS & TOTAL & DESENVOLVIDOS & SUBDESENVOLVIDOS \\
\hline Y DEFASADO & $0.0445376^{* *}$ & 2.942123 & $-0.1920023^{* * *}$ \\
& $(0.0130718)$ & $(2.250578)$ & $(0.0488275)$
\end{tabular}




\begin{tabular}{|c|c|c|c|}
\hline ÍNDICE DE GINI & $\begin{array}{c}-3.977519 * * * \\
(0.195979)\end{array}$ & $\begin{array}{c}115.5979 \\
(244.9606)\end{array}$ & $\begin{array}{c}-3.094009 * * * \\
(0.4174453)\end{array}$ \\
\hline IT/PIB & $\begin{array}{c}3.62 \mathrm{e}-08 * * * \\
(5.07 \mathrm{e}-09)\end{array}$ & $\begin{array}{c}-6.11 \mathrm{e}-07 \\
(0.4 .13 \mathrm{e}-07)\end{array}$ & $\begin{array}{c}2.33 \mathrm{e}-08 * * \\
(7.82 \mathrm{e}-09)\end{array}$ \\
\hline$T A X A A L F$ & $\begin{array}{c}0.0281261 * * * \\
(0.003722)\end{array}$ & $\begin{array}{c}0.5435349 \\
(0.4288458)\end{array}$ & $\begin{array}{c}-0.0113062 * * \\
(0.0053437)\end{array}$ \\
\hline RENDA & $\begin{array}{c}0.0028881 * * * \\
(0.0000515)\end{array}$ & $\begin{array}{l}-0.0054649 \\
(0.0049542)\end{array}$ & $\begin{array}{c}0.004503 * * * \\
(0.0001927)\end{array}$ \\
\hline $\mathrm{ABC}$ & $\begin{array}{c}0.0353928 \\
(0.1100714)\end{array}$ & $\begin{array}{c}71844.08 \\
(71844.08)\end{array}$ & $\begin{array}{c}0.0001927 \\
(0.1115365\end{array}$ \\
\hline CONSTANTE & 0.7306614 & -144.543 & 3.026914 \\
\hline \multicolumn{4}{|c|}{ Teste de Consistência do System-GMM } \\
\hline $\operatorname{AR}(2)$ & 0.3542 & 0.9449 & 0.3759 \\
\hline TESTE DE SARGAN & 21.79513 & $7.42 \mathrm{e}-16$ & 18.49638 \\
\hline
\end{tabular}

Para o país como um todo, os resultados da Tabela 3 denotam que a relação entre desigualdade de renda $\mathrm{e}$ crescimento econômico é negativa e estatisticamente significativa. Com relação às demais variáveis de controle, o investimento total como proporção do PIB (IT/PIB), a taxa de alfabetização, a renda média e a taxa de crescimento do PIB per capita defasada apresentaram relação positiva com a taxa de crescimento do PIB per capita e também foram estatisticamente significativas.

Com relação à consistência do estimador System GMM, através do Teste de Sargan verifica-se que a hipótese nula não foi rejeitada, e, assim, os instrumentos considerados são válidos. Além disso, por meio do teste de autocorrelação, AR (2), não há autocorrelação do termo de erro em todos os modelos estimados.

No que tange o principal resultado do presente estudo, que denota relação negativa entre desigualdade de renda e crescimento econômico, a literatura possui algumas explicações. Uma dessas se materializa através da restrição de crédito (HELPMAN, 2004; TACHIBANAKI,2005, WEIL， 2005). Nesse aspecto, com as imperfeições no mercado de crédito, os agentes mais pobres sofrem elevada limitação no acesso a financiamento de atividades que podem ter maior lucro. Com a referida dificuldade dessa parcela da população, tem-se um nível de produção abaixo do potencial da economia.

Outra hipótese levantada pela literatura advém da abordagem apresentada pela economia política (ALESINA, RODRICK,1994; PERSSON, TABELLINI,1994). A ideia é de que em um país com elevada desigualdade, o eleitor mediano, que não detém ativos, se sente mais propenso a votar em partidos políticos que possuam programas de redistribuição de renda, com o intuito de obter ganhos no curto prazo. No entanto, se os programas de redistribuição de renda forem realizados com base no aumento da carga tributária sobre o retorno dos investimentos, o resultado esperado poderia ser uma redução do ritmo de crescimento econômico.

Além disso, outra possibilidade discutida nos trabalhos diz respeito à instabilidade política e social, que pode impactar sobre os direitos de propriedade e desencorajar os investimentos, levando assim a uma relação negativa entre desigualdade de renda e crescimento econômico (ALESINA; PEROTTI,2016).

Adicionalmente, os resultados que levam em consideração a divisão dos estados e Distrito Federal por grupos de 
acordo com os níveis de desenvolvimento mostram claras diferenças. Primeiro, apesar da relação entre crescimento econômico e desigualdade de renda nos estados mais desenvolvidos ser positiva, esta não foi estatisticamente significativa. Nesse caso, uma possível explicação para a não significância estatística se refere ao número reduzido de estados que fazem parte do referido grupo, o que reduz em demasiado a amostra. Por outro lado, para o grupo de países menos desenvolvidos, os resultados da Tabela 3 demonstraram que a relação entre desigualdade de renda e crescimento econômico é negativa e estatisticamente significativa.

A relação entre o crescimento econômico e a desigualdade de renda no Brasil e para os grupos de estados divididos pelo seu grau de desenvolvimento apresentada no presente estudo sustenta, em parte, os resultados apontados por Barro (2000) e Shin (2012). Como destacado pelos autores, nos países pobres, a desigualdade de renda desincentiva o crescimento econômico. Por sua vez, nos países ricos, a desigualdade de renda estimula o crescimento econômico. Nesse sentido, os resultados do presente estudo sustentam que nos estados menos desenvolvidos do Brasil, a desigualdade de renda pode ser associada ao retardamento do ritmo de crescimento econômico.

Ademais, ao analisar o país durante o período 2011-2015, o estudo de Saad et al. (2020) mostrou que houve redução do índice de Gini e que as fontes de renda contribuíram para esse resultado, quais sejam: renda proveniente do trabalho, renda advinda dos programas sociais, juros de aplicação, e contribuição da aposentadoria. Conforme mencionado pelos autores, as políticas de transferência de renda no Brasil se tornaram mais relevantes com a criação do Programa Bolsa Família em 2003, o qual consiste no repasse de recursos financeiros para as famílias com renda extremamente baixa, a fim de possibilitar a inserção de seus beneficiários na sociedade. Saad et al. (2020) também apontaram que apesar dos programas de transferência de renda terem apresentado efeito positivo sobre a redução da desigualdade em todos os estados brasileiros, estes possuíram maior efeito no Norte e Nordeste do país.

Outras variáveis de controle também se mostraram associadas ao crescimento econômico. A taxa de alfabetização apresentou-se positivamente associada com uma elevação da taxa de crescimento do PIB per capita brasileiro e para o grupo de estados menos desenvolvidos. De Souza (1999) afirma que com o processo de globalização e inserção em novos mercados, seria necessário um novo perfil do trabalhador, com educação permanente e atualizada, para gerar habilidades no ambiente de trabalho. Nesse sentido, investimentos no país destinados à acumulação de capital humano possibilitam impulso no ritmo de crescimento econômico, com destaque para aqueles na área de educação e a saúde (IBGE, 2012). Como sustentado por Aghion et al. (2009), investimentos na área de educação possuem como principal retorno a capacitação dos indivíduos.

A relação positiva entre a razão dos investimentos públicos/PIB com $\mathrm{O}$ crescimento econômico do país e dos estados menos desenvolvidos sustenta o que foi verificado na literatura. $\mathrm{O}$ referido resultado é similar àqueles encontrados por Aschauer (1989), Easterly e Rebelo (1993), Devarajan et al. (1996), Pose, Tselios e Pycharis (2012), e Palei (2015). Nesse sentido, muitos desses trabalhos confirmaram a preponderância dos investimentos públicos em infraestrutura, classificando-os em produtivos e improdutivos, assim como sinalizando seus efeitos sobre o setor privado. Trabalhos aplicados ao caso brasileiro também demonstraram a existência de associação positiva entre investimentos do governo e crescimento econômico (FERREIRA, MALLIAGROS, 1998; ROCHA, GIUBERTI, 2007; BERTUSSI, ELLERY JUNIOR, 2012). 
O aumento da renda média domiciliar também apresentou associação positiva com o crescimento econômico do país como um todo e do grupo de estados menos desenvolvidos. De fato, acréscimos na renda média domiciliar da população brasileira podem sinalizar elevação do poder de compra, se estendendo a diversas atividades econômicas, o que possibilita aumento do PIB tanto do Brasil quanto dos estados menos desenvolvidos. Como demonstrado no estudo de Santos e Vieira (2016), aplicado à região Nordeste do Brasil no período 2003-2012, a renda domiciliar per capita permitiu redução da pobreza e indigência.

O termo dinâmico do modelo, representado pela taxa de crescimento anual do PIB per capita defasada em um período, mostrou relações distintas para o Brasil como um todo e para o grupo de estados menos desenvolvidos. Para o país como um todo, a taxa de crescimento do produto per capita passado influencia de forma positiva a taxa de crescimento do PIB per capita atual, e relação contrária foi observada para o grupo de estados menos desenvolvidos.

O presente estudo também inseriu a variável de controle referente ao grau de abertura comercial para controlar o efeito que as relações comerciais externas do Brasil e regiões possuíam no crescimento econômico. Nesse sentido, como salientado por Edward (1993), o comércio internacional permite transferir inovações tecnológicas, além de que maior abertura comercial eleva a concorrência no mercado local, e como resultado, tem-se aumento da eficiência da produção e do crescimento econômico (BEATON et al., 2017). No entanto, para este trabalho, essa variável não foi estatisticamente significativa, apesar do coeficiente ter sido positivo em todos os modelos.

\section{CONSIDERAÇÕES FINAIS}

Este estudo analisou a relação entre a desigualdade de renda e crescimento econômico no Brasil, utilizando informações de suas vinte e seis unidades federativas e do Distrito Federal no período 1997-2017 através de um painel dinâmico. Em particular, o trabalho investigou a relação intertemporal que existe entre essas variáveis, e em seguida verificou se a desigualdade de renda associa-se de forma positiva ou negativa ao crescimento econômico. Além disso, essa análise se estendeu para a desigualdade interestadual, levando em consideração a divisão dos estados em dois grupos: mais desenvolvidos e menos desenvolvidos.

Os resultados apontam que para o país como um todo existe uma relação bicausal entre desigualdade de renda e crescimento econômico, controlada posteriormente através da utilização do modelo com dados em painel dinâmico. Essa relação se mantém para os estados menos desenvolvidos, mas para aqueles mais desenvolvidos apenas a desigualdade de renda precede o crescimento econômico.

Além disso, os resultados também denotam que a desigualdade de renda se associa com uma redução do crescimento econômico brasileiro, e especificamente para o grupo de estados menos desenvolvidos. Diversos mecanismos, salientados pelos autores e apresentados na segunda seção, podem explicar essa relação, tal como o fato de que a parcela da população mais pobre tenha mais dificuldade de acesso a crédito, diminuindo sua capacidade produtiva, assim como do país e dos estados analisados. Adicionalmente, em um país com elevada desigualdade de renda, os eleitores, sem rendas ou ativos, possuem mais incentivo no momento de uma eleição em votar nos partidos que possuam políticas de redistribuição de renda, que quando associadas ao aumento da carga tributária no longo prazo podem reduzir o ritmo de crescimento econômico. Cabe ainda salientar que a elevada desigualdade de renda pode gerar instabilidade política, que tende a impactar nos direitos de propriedade, desencorajando os 
investimentos, o que também ocorre quando a criminalidade aumenta.

Assim, considerando o Brasil, sobretudo seus estados menos desenvolvidos, os resultados se configuram como um argumento a favor dos programas de transferências de renda, que poderiam diminuir a desigualdade de renda e garantir taxas mais elevadas de crescimento econômico. Como a desigualdade de renda é um importante determinante da pobreza, pesquisas futuras são necessárias para verificar outras consequências de sua elevação no intuito de auxiliar na promoção de políticas direcionadas à sua diminuição.

\section{REFERÊNCIAS}

ACEMOGLU, D. Matching, heterogeneity, and the evolution of income distribution, Journal of Economic Growth, v. 2, n.1, p. 61-92, 1997.

AGHION, P.; CAROLI, E.; GARCIAPENALOSA, C. Inequality and economic growth: the perspective of the new growth theories. Journal of Economic

Literature, v. 37, n.4, p.1.615-1.660, 1999.

AGHION, P.; HOWITT, P. Endogenous growth theory, MIT Press, Cambridge, 1998.

ALESINA, A.; RODRIK, D. Distributive politics and economic growth, Quarterly Journal of Economics, v. 109, n.2, p. 465490,1994.

ALESINA, A.; PEROTTI, R. Income distribution, political instability, and investment, European Economic Review, v. 40, n. 6, p. 1203-1228, 1996.

AMOS, O.M. Unbalanced regional growth and regional income inequality in the latter stages of development, Regional Science and Urban Economics, v. 18, n.4, p. 549566,1988 .
ARELLANO, M.; BOND, S.R. Some test of specification for panel data: Monte Carlo evidence and an application to employment equations. Review of Economic Studies, v. 58, n.2, p. 277-297, 1991.

ARELLANO, M.; BOVER, O. Another look at the instrumental variable estimation of error-components models. Journal of Econometrics, v. 68, n.1, p. 29-51, 1995.

ASCHAUER, D. A. Is public expenditure productive? Journal of Monetary

Economics, v. 23, n.2, p. 177- 200, March 1989.

BALASSA, B. Export incentives and export performance in developing countries: A comparative analysis, Review of World Economics, v. 114, n.1, p. 2461, March 1978.

BANERJEE, A.; DUFLO, E. Inequality and Growth: what can the data say?

Journal of Economic Growth, v. 8, n.3, p. 267-299,2003.

BARRO, R. Inequality and growth in a panel of countries. Journal of economic growth, v. 5, p. 5-32, 2000.

BARROS, R.P.; FOGUEL, M.N; ULYSSEA, G. Desigualdade de renda no Brasil: uma análise da queda recente, v. 1, Brasília: IPEA, 2006.

BEATON, K.; CEBOTARI, A.; KOMAROMI, A. Revisiting the link between trade, growth and inequality: lessons for Latin America and the Caribbean, IMF Working paper, WP/17/46, p. 1-42, 2017.

BENGOA, M.; SANCHEZ-ROBLES, B.; Does equality reduce growth? Some empirical evidence. Applied Economics Letters, v. 12, n.8, p.479-483, June 2005. 
BERNANKE, B.S.; ROTEMBERG, J. (Eds.). NBER Macroeconomics Annual, MIT Press, Cambridge, 1996.

BERTUSSI, G. L.; JUNIOR, R. E. Infraestrutura de transporte e crescimento econômico no Brasil. Journal of

Transport Literature, v. 6, n. 4, p. 101132, Oct. 2012.

BOURGUIGNON, F.J. Growth and inequality in the dual model of development: the role of demand factors, Review of Economic Studies, v. 57, n.2, p. $215-228,1990$.

BRIDA, J.G.; CARRERA, E.J.; SEGARRA, V. Clustering and regime dynamics for economic growth and income inequality. Structural Change and Economic Dynamics, v. 52, p. 99-108, March 2020.

BURANGE, L.G.; RANADIVE, R.R.;

KARNIK, N.N. Trade openness and Economic growth nexus: A case study of BRICS Foreign trade review, v. 54, n.1, p. 1-15,2019.

CASTELLÓ, A. Inequality and growth in advanced economies: an empirical investigation. The Journal of Economic Inequality, v.8, p. 293-321, 2010.

CASTRO, R.S.; JÚNIOR, S.S. Efeitos da desigualdade de renda sobre o crescimento econômico no Brasil: uma análise nãolinear. Perspectiva Econômica, v. 3, n.1, p. 27-61, jan. jun. 2007.

CHAMBERS D., KRAUSE, A. Is the relationship between inequality and growth affected by physical and human capital accumulation? The Journal of Economic Inequality, v. 8, p. 153-172, 2010.

CHEN, B.L. An inverted- U relationship between inequality and long-run growth, Economics Letters, v. 78 , n.2, p. 205212,2003 .
CRUZ, P.B.; TEIXEIRA, A.; MONTEMOR, D.S. O efeito da desigualdade da distribuição de renda no crescimento econômico. Revista Brasileira de Economia, v. 69, n.2, p. 163-186, abr. jun. 2015.

DEVARAJAN, S.; SWARROP, V.; ZOU, H.-F. The composition of public expenditure and economic growth. Journal of Monetary Economics, v. 37, p. 313-344, 1996.

DODARO, S. Comparative advantage, trade and growth: Export-led growth revisited. World development, v.19, n.9, p. 1153-1165, Sept. 1991.

DUMITRESCU, E; HURLIN, C. Testing for Granger non-causality in heterogeneous panels, Economic Modelling, v.29, n.4, p. 1450-1460, 2012.

EASTERLY, W.; REBELO, S. Fiscal policy and economic growth: an empirical investigation. Journal of Monetary

Economics. v. 32, p. 417-458, 1993.

EDWARDS, S. Openness, trade liberalization, and growth in developing countries. Journal of Economic Literature, v. 31, n.3, p. 1358-1393, 1993.

FAO, 2020. Available at: http://www.fao.org/sustainabledevelopment-goals/goals/goal-10/en/. Accessed 01/05/2020.

FERREIRA, P. C.; MALLIAGROS, T. G. Impactos Produtivos da Infraestrutura no Brasil - 1950/95. Pesquisa e

Planejamento Econômico. v. 28, n.2, p. 315-338, 1998.

FORBES, K. J. A reassessment of the relationship between inequality and growth. American Economic Review, v.90, n.4, p. 869-887, 2000. 
HELPMAN, E. The Mystery of

Economic Growth. Belknap Harvard, 2004.

HERRERIAS, M.J.; ORTS, V. Is the export-led growth hypothesis enough to account for China's growth? China \& World Economy, v.18, n.4, p.34-51, 2010.

HERZER, D.; VOLLMER, S. Inequality and growth: evidence from panel cointegration. The Journal of Economic Inequality, v.10, n.4, p. 489-503, 2012.

IBGE- Instituto Brasileiro de Geografia e Estatística. Available at: https://agenciadenoticias.ibge.gov.br/agencia -noticias/2012-agencia-denoticias/noticias/27596-nordeste-e-unicaregiao-com-aumento-na-concentracao-derenda-em-2019. Accessed: 25/25/2020.

KNOWLES, S. Inequality and economic growth: The empirical relationship reconsidered in the light of comparable data. J. Dev. Stud., v. 41, n.1, p. 135$139,2005$.

KONG, Q.; PENG, D.; JIANG, X.; WANG, Z. Trade openness and economic growth quality of China: Empirical analysis using ARDL model. Finance Research Letters, March 2020.

LI, H.; ZOU, H. Income inequality is not harmful for growth: theory and evidence, Review of Development Economics, v. 2, n.3, p. 318-334,1998.

MURPHY, K.M.; Shleifer, A.; Vishny, R. Income distribution, market size, and industrialization, Quarterly Journal of Economics, v. 104, n.3, p. 537-564, 1989.

OHANA. Available:

https://www.cartacapital.com.br/sociedade/i bge-27-das-familias-ganham-um-quinto-detoda-a-renda-no-brasil/. Accessed 01/05/2020.
OKUN, A.M. Equality and Efficiency: the Big Trade-Off. Brookings Institution, Washington, DC, 1975.

PALEI, T. Assessing the impact of infrastructure on economic growth and global competitiveness. Procedia Economics and Finance, v. 23, p. 168$175,2015$.

PANIZZA, U. Income inequality and economic growth: Evidence from American data. Journal of Economic Growth, v. 7, n.1.p. 25-41, 2002.

PEROTTI, R. Growth, income distribution, and democracy: what the data say. Journal of Economic Growth, v.1, n.2, p. 149187, 1996.

PEROTTI, R. Political equilibrium, income distribution, and growth. Review of Economic Studies, v.60, n.4, p. 755776, 1993.

PERSSON, T.; TABELLINI, G. Is inequality harmful for growth? American Economic Review, v.84, n.3, p. 600621,1994 .

POSE, A. R.; PSYCHARIS, Y.; TSELIOS; $\mathrm{V}$. Public investment and regional growth and convergence: Evidence from Greece. Papers in Regional Science, v. 91, n. 3, Aug. 2012.

ROCHA, F.; GIUBERTI, A. C. Composição do Gasto Público e Crescimento Econômico: um estudo em painel para os estados brasileiros.

Economia Aplicada, São Paulo, v. 11, n. 4, p. 463-485, out.-dez. 2007.

SAAD, M.G.; BORTOLUZZO, A.B.; FILHO, N.A.M.; KOMATSU, B.K. Os determinantes da queda da desigualdade de renda nas regiões brasileiras entre $2001 \mathrm{e}$ 2015. Revista Brasileira de Ciências Sociais, v. 35, n.104, 2020. 
SANTOS, M.P.; CUNHA, M.S.;

GADELHA, S.R. Distribuição de renda e crescimento econômico no Brasil: Uma análise econométrica do período 19762010. Revista de Economia, v. 43, n.1, jan. abr.2016.

SANTOS, V. F.; VIEIRA, W.C. Crescimento econômico e desigualdade de renda: uma análise sobre pobreza e extrema pobreza na região Nordeste do Brasil. Indicadores Econômicos, v. 44, n.2,2016.

SATTI, S.L.; HASSAN, M.S.; HAYAT, F.; PARAMATI, S.R. Economic Growth and inflow of remittances: Do they combat poverty in an emerging economy? Social Indicators Research, v.127, p.1119-1134, 2016.

SHIN, I. Income inequality and economic growth, Economic Modelling, v.29, n.5, p. 2049-2057, set. 2012.

SHIN, I.; KIM, H.; YAMAMURA, J.E. Technological Progress and the Future of Kuznets Curve's, MPRA (Munich Personal RePEc Archive) Paper no. 18866 (2009).
SOUZA, H.G. Efeitos do crescimento econômico e da desigualdade de renda sobre a pobreza no Brasil. Economía, sociedad y territorio, v.19, n.60, 2019.

SUKIASSYAN, G. Inequality and growth: what does the transition economy data say? Journal of Comparative Economics, v.35, n.1, p. 35-56, 2007.

TACHIBANAKI, T. Confronting income inequality in Japan - a comparative analysis of causes, consequences, and reform, MIT Press, 2005.

VOITCHOVSKY, S. Does the profile of economic inequality matter for economic growth? Distinguishing between the effects of inequality in different parts of the income distribution. Journal of economic growth, v. 10, p. 273-296, 2005.

Weil, D. N. Economic Growth, AddisonWesley, 2005.

WOOLDRIDGE, J. Econometric analysis of cross section and panel data. 2. ed. Cambridge: MIT Press, 2010.

WORLDBANK,2020.

https://www.worldbank.org/en/topic/isp/ov erview\#2. Accessed: 20 maio 2020.

\footnotetext{
${ }^{\mathrm{i}}$ Graduação em Ciências Econômicas com ênfase em Agronegócio pela Universidade Federal de Viçosa (2016), Mestra em Economia Aplicada (2018), e atualmente cursa doutorado em Economia Aplicada (DER) da mesma instituição

ii Doutorado em Economia Aplicada pela ESALQ/USP, mestrado em Desenvolvimento Econômico pela Universidade Federal do Paraná (UFPR) e graduação em Economia pela Universidade Federal de Juiz de Fora (UFJF)
} 